\title{
PERCEPCIONES DE LAS TUTORÍAS DE ESCRITURA EN UN CENTRO NOVEL
}

\section{WRITING TUTORING PERCEPTIONS IN A NOVEL CENTER}

\author{
Génesis Caridad Granados Ayala \\ Universidad de Montemorelos, México \\ granadosgenesis@um.edu.mx \\ https://orcid.org/0000-0002-8753-4456
}

\begin{abstract}
RESUMEN
Esta investigación buscó conocer las percepciones, estrategias y retos de las tutorías de escritura implementadas en una universidad privada mexicana. El enfoque fue cualitativo, con diseño de estudio de caso y se realizó una triangulación de los instrumentos utilizados (observación, entrevistas, documentos, literatura, investigador). Según los resultados, se perciben mejorías en la escritura y un ambiente de confianza en las tutorías. Las estrategias utilizadas incluyen la lectura en voz alta y las preguntas, y los principales retos son la falta de tiempo, la brecha generacional y la difusión.
\end{abstract}

Palabras clave: enseñanza de la escritura, tutoría, enseñanza superior

\section{ABSTRACT}

This research sought to know the perceptions, strategies, and challenges of the tutorials implemented in a recently created writing center in a private Mexican university. The approach was qualitative, with a case study design and a triangulation of the instruments used (observation, interviews, documents, literature, researcher). According to the results, improvements are perceived in writing and an atmosphere of trust towards the tutorials. The strategies used include reading aloud and asking questions, and the main challenges are lack of time, generation gap, and outreach.

Keywords: writing teaching, tutoring, higher education, writing centers

\section{Introducción}

La universidad no solamente tiene como responsabilidad transmitir conocimientos, sino que necesita preparar a los estudiantes para las situaciones futuras y las necesidades sociales. La sociedad actual demanda productores de conocimientos que puedan transmitir lo descubierto
(Moyano, 2010). Sin embargo, esto no será posible si los estudiantes no saben escribir. Si no se prepara a los futuros profesionistas en esta competencia, se los incapacitará en su formación académica, en su búsqueda laboral y en su desempeño profesional, repercutiendo también en su participación social (Flores Guerrero, 2016). 
La redacción de textos complejos y pertinentes es uno de los principales retos que enfrentan los estudiantes universitarios en su formación (Castro Azuara y Sánchez Camargo, 2015). La escritura representa no solo un fin de las competencias de egreso de los estudiantes de pregrado, sino un medio para la adquisición de otras competencias (Giraldo Giraldo, 2015). Existe evidencia de que los alumnos que participaron en programas de acompañamiento de escritura mejoraron también sus estrategias de aprendizaje y, a su vez, su rendimiento académico (González Jaimes et al., 2013).

Diversas instituciones educativas han creado programas que se ajustan a las necesidades y a las limitaciones de cada institución (Molina Natera, 2015). Entre estos programas se destacan los centros de escritura, los programas de acompañamiento (Calle Álvarez, 2014) y la implementación de la escritura a través del currículo (Carlino, 2004).

Los centros de escritura son espacios cuyo principal objetivo es apoyar, por medio de tutorías de escritura, la producción escrita de los alumnos que tienen dificultades especialmente en la universidad (Calle Arango et al., 2017; Chois-Lenis et al., 2017; Molina Natera, 2014). A partir de septiembre de 2019 se implementó un centro de escritura en la institución donde se desarrolló el presente estudio. El propósito de esta investigación fue observar su desarrollo durante el primer año de funcionamiento, junto con las estrategias aplicadas y los retos identificados a partir de las percepciones de sus actores.

\section{Referentes conceptuales}

Escritura. Cassany (1995) define la escritura como la capacidad de expresar información de forma coherente y co- rrecta con la finalidad de que otras personas la entiendan. Por su parte, Ballesteros Pérez (2016) afirma que esta "permite la construcción de la propia individualidad, la identidad, el conocimiento y el sentido de pertinencia" (p. 448).

Cassany (1993) identificó tres dimensiones de la escritura: los conocimientos, las habilidades y las actitudes. Estas se describen a continuación.

Conocimientos: para que un escrito tenga éxito, debe tener (a) adecuación sobre el nivel de formalidad requerido, (b) estructura y coherencia, (c) cohesión, concordancia y puntuación, (d) gramática y ortografía, (e) presentación del texto y (f) recursos retóricos.

Habilidades: además de los conocimientos, escribir requiere poner en marcha habilidades cognitivas, tales como (a) análisis, (b) formulación de ideas, (c) esquematización, (d) planeación, (e) valoración del texto y (d) reescritura.

Actitudes: las actitudes son muy relevantes para el desarrollo de la escritura, incluso más que las habilidades y los conocimientos. De las actitudes dependen la motivación, el interés, el placer o el aburrimiento frente a la escritura.

Cassany (1994) identificó también tres aspectos cognitivos que conforman los pasos de la escritura: la planificación, la redacción y la revisión. Otros autores agregan el de la reescritura, en donde se busca que el texto se reescriba y resulte mucho mejor que el inicial.

Aunque la escritura involucra una serie de procesos mentales y la ejecución de diversas habilidades cognitivas, los estudiantes regularmente utilizan la escritura solo como un instrumento para cumplir con tareas académicas, lo que suele impedir que desplieguen competencias de escritura de alto nivel (Guzmán-Simón y García-Jiménez, 2014). 
Tutorías de escritura. Las tutorías de escritura constituyen un acompañamiento para los estudiantes que les puede dar sugerencias para mejorar sus escritos (Calle Álvarez, 2014) y crear escritores independientes (Molina Natera, 2015). Según Molina Natera (2014), "son una forma de trabajo colaborativo para el desarrollo de habilidades de escritura" (p. 9), lo cual coincide con Hobson (1992), quien afirmó que se basan en el constructivismo social, pues la escritura se logra mediante la interacción y el trabajo colaborativo.

Una de las premisas básicas del constructivismo es que el aprendizaje requiere desarrollarse en un entorno social (Schunk, 2012). Asimismo, la escritura es una práctica social que resulta ser un medio y un fin en la comunicación, la interacción y la construcción del aprendizaje. Aguirre Seura (2016) encontró que quienes participan en programas de escritura mejoran sus habilidades metacognitivas.

Teoría sociocultural de Vygotsky. Según Vygotsky (1995), el entorno social y cultural propicia el aprendizaje significativo, así como el dominio del lenguaje escrito y la lectura, lo cual permite acceder a todo el conocimiento escrito que el ser humano ha creado. Es decir, "el lenguaje escrito es el instrumento para la construcción del aprendizaje y la autorregulación del conocimiento" (p. 476).

En su teoría sociocultural, Vygotsky creó el concepto zona de desarrollo próximo, en el que explica que existe una distancia entre lo que el alumno sabe y puede hacer por sí mismo y lo que puede saber y hacer con la ayuda de alguien con más experiencia, ya sea un adulto o un par. Estas aproximaciones fomentan su desarrollo cognoscitivo.
De allí derivan las tutorías entre pares, en donde se eligen pares de un mismo contexto social y se asigna al individuo que tiene mejor nivel para ejercer el papel de tutor. En el caso de la escritura, los más experimentados deben construir andamiajes que propicien la interacción y el desarrollo de esta competencia en los inexpertos, convirtiéndose en mediadores del aprendizaje (Ballesteros Pérez, 2016).

Esta aproximación busca eliminar las relaciones autoritarias tradicionales entre el estudiante y el profesor, pues al sentirse como iguales se propician retroalimentaciones recíprocas, lo que facilita la producción de conocimientos (Calle Arango et al., 2017).

\section{Teoría del desarrollo cognoscitivo.} Piaget propuso cuatro etapas del desarrollo cognoscitivo: sensoriomotriz, preoperacional, operacional concreta y operación formal. La última etapa comienza en la adolescencia y se extiende hasta la edad adulta. En ella, el pensamiento es más lógico y deductivo y los individuos pueden crear múltiples soluciones a problemas, con un pensamiento científico, y son capaces de comprender ideas más abstractas.

La escritura implica una serie de procesos mentales y la ejecución de diversas habilidades cognitivas superiores. Por lo tanto, los individuos pueden desarrollar plenamente esta competencia al llegar a la cuarta etapa del desarrollo cognoscitivo. Sin embargo, para desarrollar un aprendizaje significativo se requiere de la interacción con los demás (Piaget, 1972), mediante un proceso en el que el docente pase de ser el protagonista a ser un guía en el proceso de construcción social del conocimiento (Calle Arango et al., 2017). 
En síntesis, las tutorías de escritura se fundamentan en la teoría sociocultural de Vygotsky y en la teoría del desarrollo cognoscitivo de Piaget, cuyas premisas se basan en el logro del aprendizaje gracias al entorno social, al trabajo colaborativo, al acompañamiento de alguien con mayor experiencia y al logro del nivel cognoscitivo necesario para comprender las estructuras complejas de la escritura.

\section{Metodología}

Se utilizó un enfoque cualitativo, ya que este enfoque busca consolidar e interpretar la información que los participantes aportan y la información que el investigador ha observado y leído (Merriam, 1998). El estudio de caso único busca discutir y explicar lo que sucedió para entender el fenómeno, razón por la cual se decidió utilizar este diseño.

Participantes. Se eligieron cuatro alumnos, tres de los cuales asistieron al centro por iniciativa propia para la revisión de proyectos de escritura y uno por derivación, para la revisión de un ensayo. Asimismo, se eligieron cuatro tutores: dos de experiencia y dos pares. Los tutores entrevistados fueron los mismos que atendieron a los alumnos seleccionados.

Triangulación de los instrumentos. Cualquiera que sea la forma para realizar la triangulación, "aumenta la credibilidad y la calidad" del estudio (Patton, 2015, p. 674). Para lograr credibilidad en esta investigación, se recolectaron datos de diversas fuentes con el propósito de responder a las preguntas que guiaron este estudio (Merriam y Tisdell, 2016). Estas fuentes fueron la literatura, las entrevistas, la observación, el análisis de documentos y la interpretación del investigador (ver Tabla 1).

\section{Tabla 1}

Matriz de triangulación en relación con las preguntas de investigación con las fuentes de datos

\begin{tabular}{|c|c|c|c|c|c|}
\hline Preguntas de investigación & $\begin{array}{c}\text { Revisión } \\
\text { de la } \\
\text { literatura }\end{array}$ & Entrevistas & $\begin{array}{c}\text { Registro de } \\
\text { observación }\end{array}$ & $\begin{array}{c}\text { Análisis de } \\
\text { documento }\end{array}$ & $\begin{array}{c}\text { Interpre- } \\
\text { tación del } \\
\text { investigador }\end{array}$ \\
\hline $\begin{array}{l}\text { 1. ¿Cuáles son las necesidades } \\
\text { iniciales por las que los } \\
\text { alumnos acudieron a las } \\
\text { tutorías? }\end{array}$ & $\checkmark$ & $\checkmark$ & $\checkmark$ & $\checkmark$ & $\checkmark$ \\
\hline $\begin{array}{l}\text { 2. ¿Qué estrategias utilizaron } \\
\text { los tutores para asistir las } \\
\text { necesidades iniciales de } \\
\text { escritura de los alumnos? }\end{array}$ & $\checkmark$ & $\checkmark$ & $\checkmark$ & $\checkmark$ \\
\hline $\begin{array}{l}\text { 3. ¿Cuáles son las valoraciones } \\
\text { de los alumnos y tutores } \\
\text { respecto a las tutorías de } \\
\text { escritura? }\end{array}$ & $\checkmark$ & $\checkmark$ & $\checkmark$ & $\checkmark$ \\
\hline $\begin{array}{l}\text { 4. Según los hallazgos, ¿cuáles } \\
\text { serían las posibles mejoras } \\
\text { para las tutorías de escritura? }\end{array}$ & $\checkmark$ & $\checkmark$ & $\checkmark$ & \\
\hline
\end{tabular}


Análisis de datos. Se transcribieron las grabaciones de las ocho entrevistas y se importaron al programa de análisis cualitativo ATLAS.ti (versión 8.4.4.). Se identificaron 17 códigos, a los cuales se les asignó un nombre por cada vez que un concepto o idea se repitió tres o más veces y se agruparon esos códigos en cuatro categorías. Después, se procedió a realizar el análisis de los hallazgos y se realizó una triangulación para conferir confiabilidad a los resultados.

Confiabilidad. Para garantizar la confiabilidad del presente estudio, se buscó la credibilidad, la consistencia, la transferibilidad y la confirmabilidad.

Para asegurar la credibilidad de este estudio y enfatizar los hallazgos, haciéndolos creíbles para el investigador, los participantes y la audiencia (Merriam, 2009), se utilizó la triangulación de las fuentes de datos y la revisión de los resultados por parte de pares.

Para la consistencia de los resultados, se utilizó la triangulación por medio de la integración de la postura del investigador, la observación, las entrevistas, el análisis de los documentos y la revisión de la literatura. Se resguardaron de manera fidedigna las bases de información obtenidas.

Respecto a la transferibilidad, para los casos como el de este estudio, las investigaciones afirman que las tutorías de escritura no son replicables, puesto que son una atención personalizada para atender las necesidades de escritura de los alumnos en contextos específicos (Molina Natera, 2019a). Sin embargo, el lector decidirá si los pasos que se siguieron para lograr los objetivos en esta investigación pueden transferirse a otros contextos.

Además de hacer referencia a los estudios y hallazgos de otros autores, para lograr la confirmabilidad en este estudio, se atendieron las cuestiones éticas. Los participantes fueron ilustrados sobre los propósitos del presente estudio y se les pidió firmar una carta de consentimiento informado de acuerdo con la "Ley de Protección de Datos en Posesión de Particulares" (Diario Oficial de la Federación, 2016) si decidían formar parte de él.

\section{Implementación de la estrategia}

Diagnóstico. A inicios del año 2019, se creó un instrumento para diagnosticar la competencia de escritura y se lo administró a 234 alumnos ingresantes a la universidad. En la prueba correspondiente al área de escritura, que constaba de 30 reactivos con un rango de valores posibles de 0 a 30 puntos, se obtuvo una media de 16 puntos. Este resultado mostró que el nivel general de los alumnos en la competencia de escritura no era alto y, por lo tanto, se requerían acciones para nivelar esas deficiencias. $\quad \mathrm{L} \mathrm{o} \mathrm{s}$ resultados de este diagnóstico fueron comunicados a los directores de las facultades con la finalidad de que derivaran al centro de escritura a los alumnos que obtuvieron los puntajes más bajos.

Organización. Para el diseño e implementación del centro de escritura, se siguieron las orientaciones sugeridas por la literatura. Además, se visitó un centro de escritura ya establecido y se asistió a congresos para recuperar información que fuera de beneficio para la puesta en marcha de este proyecto.

Se realizó una invitación personal a cada uno de los tutores de escritura, quienes aceptaron participar de manera voluntaria. En total se integraron al equipo dos maestros jubilados, cinco maestros de la planilla de empleados de 
la universidad y tres alumnos con habilidades destacables de escritura. Una vez integrado el equipo de tutores, se llevó a cabo una reunión general para familiarizarlos con los objetivos de las tutorías de escritura. Se les informó que habría reuniones quincenales de capacitación y seguimiento en donde también podrían compartir sus inquietudes y experiencias. Posteriormente, se ofreció el servicio de las tutorías de escritura a los alumnos y docentes universitarios.

Intervención. Al iniciar la tutoría, el tutor le administraba al alumno una prueba diagnóstica y después revisaban juntos el texto para el que se requería ayuda. Finalmente, se invitaba al alumno a programar otra cita y, una vez que este se retiraba, el tutor registraba las dificultades iniciales del alumno y las estrategias que había utilizado en la asesoría.

Se esperaba que los alumnos asistieran varias veces, de manera que, cuando sintieran resueltas sus necesidades, se les aplicara una prueba equivalente a la inicial para medir sus avances. Sin embargo, los alumnos dejaban de ir a sus asesorías, por lo que fue imposible medir longitudinalmente sus logros. Además, los registros no poseían información suficiente para saber cómo habían transcurrido las asesorías. Ante esto, se realizó y se envió una encuesta electrónica a los alumnos para saber las razones por las que dejaron de asistir, pero esta no fue contestada.

Informe. Se llevó un registro de las citas programadas y de la bitácora de la asesoría. En mayo de 2020 se encontraron 31 estudiantes registrados. Se sabe que un número mayor de alumnos utilizaron los servicios de tutoría de es- critura, pero por diversos motivos no se registraron sus visitas.

Con base en los documentos, se encontró que los alumnos solicitaron más citas con los tutores pares, aunque solo se contaba con tres de ellos. Asimismo, se encontró que la mayoría acudió por iniciativa propia y solicitó ayuda en sus proyectos de investigación y ensayos.

Sin embargo, esta información no fue suficiente para identificar, explorar y comprender desde las percepciones de los participantes (a) las necesidades iniciales de escritura de los alumnos, (b) las estrategias que utilizan los tutores y (c) los retos a los que se enfrentan las tutorías de escritura.

\section{Resultados}

Las preguntas de investigación ayudaron a guiar, organizar y orientar el presente estudio (Creswell, 2012; Merriam y Tisdell, 2016; Sagor, 2000) y la presentación de los hallazgos está basada en ellas.

En respuesta a la primera pregunta de investigación, ¿cuáles son las necesidades iniciales por las que los alumnos acudieron a las tutorías?, se encontró que los alumnos perciben que tienen necesidades en (a) la planeación, b) la redacción y c) la revisión de sus textos. Cabe destacar que los tutores descubrieron y atendieron también otras necesidades específicas de escritura de las cuales los alumnos no eran conscientes.

Respecto de la segunda pregunta de investigación, ¿qué estrategias utilizaron los tutores para asistir las necesidades iniciales de los alumnos?, se encontró que los tutores emplearon las siguientes estrategias en sus de tutorías de escritura: (a) lectura en voz alta de los textos, (b) preguntas reflexivas y proactivas 
sobre el texto, (c) retroalimentación, (d) explicación de bases gramaticales y ejemplificación sobre su uso y (e) recursos tales como esquemas de planeación, redacción y antiplagio y ejercicios y técnicas para la cohesión.

La tercera pregunta de investigación, ¿cuáles son las valoraciones de los alumnos y tutores respecto a las tutorías de escritura?, fue respondida al encontrarse que los alumnos y tutores valoran (a) la mejoría en los diferentes pasos y dimensiones de la escritura; (b) un ambiente de confianza, lo que permitió una mejor interacción entre tutores y alum- nos y una tutoría más productiva; (c) la disposición del tutor para atender las necesidades de escritura y (d) la preferencia del tutorado de un seguimiento con el mismo tutor.

Respecto de la pregunta ¿cuáles son los retos a los que se enfrentan las tutorías de escritura?, se encontró que los principales retos fueron (a) la falta de tiempo, (b) las limitaciones del tutor, (c) la brecha generacional, (d) la evaluación y (e) la difusión.

En la Tabla 2 se sintetizan los resultados de los códigos y las categorías obtenidos.

\section{Tabla 2}

Categorías y códigos emergentes del análisis de las entrevistas

\begin{tabular}{llll}
\hline $\begin{array}{c}\text { Acompañamiento } \\
\text { del tutor }\end{array}$ & Estrategias del tutor & Valoración de las tutorías & \multicolumn{1}{c}{$\begin{array}{c}\text { Retos } \\
\text { de las tutorías }\end{array}$} \\
\hline Planeación & Lectura en voz alta & Mejoría en la escritura & Falta de tiempo \\
Redacción & Preguntas & Ambiente de confianza & Limitación del tutor \\
Revisión & Retroalimentación & $\begin{array}{l}\text { Disposición del tutor } \\
\text { Beguimiento con el } \\
\text { Bases gramaticales }\end{array}$ & Evaluación \\
& Recursosional & Difusión \\
\hline
\end{tabular}

\section{Discusión de resultados}

Para esta sección, la discusión de los resultados, en donde se presenta la triangulación de la información obtenida de las diferentes fuentes, se asignaron nombres ficticios para resguardar la confidencialidad y privacidad de los participantes. Samuel (EAS) y Juan (EAJ) fueron dos alumnos asistidos por dos tutores de experiencia, Elí (ETEE) y Jesús (ETEJ), respectivamente. Ruth (EAR) y Miriam (EAM) fueron asistidas por dos tutoras pares, Noemí (ETPN) y Jocabet (ETPJ), respectivamente.
Acompañamiento del tutor. Las necesidades de escritura atendidas por los tutores fueron la planeación, la redacción y la revisión de textos. Según Cassany (1994), estos son los pasos que conforman la escritura.

Planeación. Se observó que los alumnos sentían frustración cuando se les pedía redactar un ensayo en la exploración diagnóstica, pues no sabían cómo aterrizar sus ideas ni elegir el contenido o la estructura que debían seguir. Juan mencionó que es un problema general que percibe en sus compañeros y que debe atenderse porque "se les abandona mucho" (EAJ4:27). 
Molina Natera (2015) afirma que "se ha identificado la planeación como la etapa en la que los estudiantes presentan la mayor dificultad" (p. 247), mientras que Murray (1982) expresa que esta debería tomar el mayor tiempo del proceso de escritura.

Por su parte, el tutor Elí mencionó que los maestros están fallando al asignar ciertas tareas a los alumnos, porque no les dan lineamientos de lo que esperan y los alumnos no saben cómo planear y estructurar sus escritos (ETEE 8:16).

Por lo anterior, se interpreta que los alumnos tienen dificultades en la planeación, porque no poseen las herramientas necesarias para redactar con propiedad sus textos y los procesos básicos de la escritura, pero también por la falta de instrucción de sus docentes.

Redacción. Para que cualquier escrito tenga éxito como medio de comunicación, debe tener (a) adecuación sobre el nivel de formalidad requerido, (b) estructura y coherencia, (c) cohesión, concordancia y puntuación, (d) gramática y ortografía, (e) presentación del texto y (f) recursos retóricos (Cassany, 1993). Se observó que la mayoría de los estudiantes universitarios desconocen los elementos necesarios para redactar sus textos académicos, por lo que se les dificulta recuperar y transcribir la información obtenida en fuentes de información, lo que propicia el plagio, ya sea accidental o intencional, el cual es uno de los problemas recurrentes en la escritura académica (Molina Natera, 2015). Ruth dijo: "más que nada mis dudas eran con respecto a la parte del plagio, tenía muchas dudas que nadie me había... respondido, ni siquiera en clases, y era lo que quería... aclarar" (EAR 1:3).

Según Molina Natera (2015), las tutorías de escritura deben atender las necesidades específicas de redacción de los alumnos. Noemí, por ejemplo, expresó que el texto que Ruth le mostró tenía muchos problemas (ETPN 5:3), por lo que basó su tutoría en la premisa de hacerla "una escritora independiente" y que no esperara que su tutora corrigiera su texto, sino que "se sintiera capacitada para avanzar con su proyecto de tesis" (ETPN 5:2).

Lo anterior coincide con la premisa principal de las tutorías de escritura, según la cual estas constituyen un acompañamiento que puede dar sugerencias para mejorar los textos (Calle Álvarez, 2014) y crear escritores independientes (Molina Natera, 2015).

Revisión. Se observó que, en general, los alumnos no revisan sus trabajos escritos. Cuando llegaban a la tutoría y se les pedía que leyeran su texto en voz alta, reconocían que no entendían lo que habían escrito y que era la primera vez que lo leían (EAM 2:7). Así lo admitió Juan, al decir "realmente cuando yo hacía algo, yo hacía un ensayo de la escuela, lo terminaba...y lo mandaba. Ni lo leía y, si lo leía, era así papaapa, pum, vámonos" (EAJ 4:30). Pero admitió que su tutor le enseñó la importancia de revisar sus escritos (EAJ 4:21).

Aunque algunos alumnos estuvieron de acuerdo en recibir ayuda para realizar sus revisiones de manera independiente, otros buscaban soluciones más rápidas, tal como lo expresó Jocabet respecto a su alumna, quien llegó derivada por su maestra, buscando un trabajo de edición:

Quería que ella se diera cuenta de dónde tenía su error, que al leer en su ensayo ella misma se diera cuenta de sus errores... no precisamente que yo le revisara y que le marcara sus errores, sino que ella se diera cuenta. (ETPJ 6:4) 
Los alumnos no revisan sus textos después de escribirlos (ETPJ 6:36), por lo que no saben identificar sus problemas de escritura, ni siquiera sus errores. Sin embargo, al acudir a las asesorías, los tutores expertos pueden hacerles ver que tienen otras necesidades, algunas veces más prioritarias que aquellas por las que solicitan la asesoría; por ejemplo, la revisión de sus escritos, un paso fundamental en el proceso de escritura (Cassany, 1993).

En la misma dirección, Elí, un tutor de experiencia, argumentó que los maestros no son tan exigentes con los textos, por lo que los alumnos no se autoexigen o no les interesa mejorar su escritura y no sienten la necesidad de revisar sus textos, mucho menos solicitar tutorías de escritura (ETEE 8:20). Tales afirmaciones coinciden con lo expresado por Samuel, quien mencionó que ningún maestro le ha señalado algún error de redacción a lo largo de su carrera, ni siquiera su asesora de investigación, aunque esto último lo justifica por el exceso de trabajo que implicaría revisar los escritos de todos los alumnos (EAS 3:11).

En síntesis, los alumnos solicitan asistencia en cualquiera de las tres etapas del proceso de escritura, por lo que los tutores que brindan acompañamiento deben conocer diferentes estrategias para ofrecer una tutoría eficaz (Molina Natera, 2011).

Estrategias del tutor. Se identificaron las siguientes estrategias utilizadas en las tutorías de escritura: (a) lectura en voz alta, (b) preguntas, (c) retroalimentación, (d) bases gramaticales y (e) recursos.

Lectura en voz alta. En las reuniones formativas quincenales de los tutores, se insistió en que las tutorías no pretenden editar los textos de los alumnos, por lo que una de las principales estrategias es alternar la lectura en voz alta, para que el alumno pueda identificar sus errores y los corrija por sí mismo (Molina Natera, 2015).

Se observó y se confirmó, mediante las entrevistas, que los tutores solicitaban al alumno que leyera su texto (ETPN 5:18; ETPJ 6:6; ETEJ 7:36) o le daban la opción de que fuera el tutor quien lo leyera, aunque la mayoría de los alumnos elegía leerlo por sí mismo. Juan, por su parte dijo: "normalmente leíamos juntos el texto, él leía un párrafo y yo leía otro o yo leía una línea o una... oración y él leía la siguiente. A veces yo leía todo" (EAJ 4:18). Esta estrategia le ayudaba a darse cuenta por sí mismo de sus errores, al igual que Ruth, quien expresó que gracias a la lectura del texto pudieron localizar errores como reiteración, ortografía y puntuación (EAR 1:5). Lo mismo sucedió con Miriam, quien dijo que "mientras lo íbamos leyendo, lo íbamos corrigiendo. Entonces ahí nos dimos cuenta de varias cosas" (EAM 2:5). Por su parte, Jocabet consideró que fue la mejor estrategia que usó como tutora de escritura (ETPJ 6:34) y, a su vez, Jesús, uno de los tutores de experiencia, mencionó que esta lectura le servía para darse cuenta de lo que el alumno necesitaba (ETEJ 7:36).

Preguntas. Molina Natera (2019a) señala que en las tutorías de escritura el tutor acompaña y realiza las preguntas correctas para que los alumnos lleguen a sus propias respuestas. El alumno es quien más debe hablar, pues las tutorías de escritura no son clases, sino un espacio donde el alumno es el protagonista (Calle Arango et al., 2017).

Para transferir ese tradicional protagonismo, el tutor debe saber qué 
preguntas hacer, cuándo hacerlas y hacer comprender al alumno que no hay respuestas ni preguntas preconcebidas, pues las tutorías son "una conversación de la escritura con el pretexto de un texto" (Molina Natera, 2019a), que se adapta a las necesidades individuales de los alumnos, quienes tienen la oportunidad de expresarse con libertad.

Se observó que el tutor hacía preguntas reflexivas para que el alumno identificara sus errores. Cuando el alumno no identificaba su error, el tutor lo orientaba mediante preguntas proactivas basadas en la lectura del texto. Noemí, por ejemplo, hacía preguntas como

ite parece que ese texto se escucha bien?, ¿crees que hay algo que se puede modificar? (ETPN5:17), ¿crees que hay un error?, ¿en dónde crees que está el error?, ¿qué crees que es lo que haga falta?; esta idea ¿qué tiene que ver con esta otra?, ¿es una consecuencia?, ¿es una causa? (ETPN 5:19)

Ruth, por su parte, expresó que Noemí le hacía preguntas "básicamente con relación a cómo quería ir yo desarrollando mi proyecto..." (EAR 1:14), lo cual coincide con la premisa de las tutorías de escritura que afirma que el tutor no hace el escrito, sino que el control del texto lo tiene el alumno, por lo que el tutor debe preguntar qué es lo que el alumno quiere o necesita plasmar en su texto y cómo es que quiere hacerlo (Molina Natera, 2019b). Asimismo, Jocabet explicó que hacía preguntas enfocadas al mismo propósito, pero que su alumna también le hacía preguntas (ETPJ 6:23), al igual que en el caso del tutor Jesús, quien dijo que le hacía algunas recomendaciones a su tutorado y que este preguntaba el porqué de ellas y entonces le explicaba (ETEJ 7:13). Así ambos actores interactuaban.
Retroalimentación. La interacción que hay entre el tutor y el alumno define cómo debe realizarse la retroalimentación, pues esta se ajusta al estudiante (Ferreira et al., 2012). La tutoría de Jesús está alineada con este postulado (ETEJ 7:34). Para conocer la perspectiva del alumno, se le preguntó a Juan sobre las estrategias de su tutor Jesús, a lo que contestó:

Por ejemplo, yo quería hacer algo, pero él me decía: “¿sabes qué?, vamos a trabajar mejor en esto", y probamos las dos ideas y ya quedamos en que... "¿sabes qué? esa es la mejor o esta está mejor" ... Hacíamos contrastes. Es decir que "¿sabes qué? esta expresión tuya no fue la mejor, vamos a hacerlo de esta manera y si no queda mejor, utilizamos la otra". (EAJ 4:10)

Por su parte, el tutor Elí mencionó que utilizó la retroalimentación para que los alumnos no fueran dependientes de él o de las tutorías de escritura, sino que se convirtieran en escritores autónomos (ETEE 8:13). Molina Natera (2011) afirma que "los tutores son expertos lectores que reaccionan como reaccionaría el lector o los lectores del texto y en ese sentido brindan la retroalimentación específica que requiere el tutorado" (p. 9).

La retroalimentación facilita la comunicación entre tutor y alumno y la producción de conocimientos de ambos actores, ya que no solo el alumno es el que aprende o desarrolla su conocimiento de escritura, sino que también el mismo tutor construye más aprendizajes en diferentes ámbitos (Calle Arango et al., 2017).

Bases gramaticales. Se observó que los tutores sentían la necesidad de explicar las bases gramaticales y daban 
ejemplificación de las lecciones enseñadas (EAR 1:21), pues a algunos alumnos les resultaba difícil comprenderlas y realizar la transferencia de ese conocimiento a sus textos escritos. El tutor Jesús expresó que recibió a alumnos "bastante... rezagados en cuanto al conocimiento de su lengua y... en esos casos, sí hay que ir desde lo más simple" (ETEJ 7:22), por lo que les enseñaba los rudimentos de la gramática a partir de los textos escritos por los alumnos (ETEJ 7:28). Para él, la idea de las tutorías de escritura es avanzar y dejar "bien pulida" la redacción y los conocimientos de gramática de los alumnos para que después puedan trabajar solos (ETEJ7:20). Molina Natera (2019a) señaló que en las tutorías no se ofrece contenido ni clase de gramática; sin embargo, en este estudio se observó la necesidad de dedicar cierto momento de la tutoría para compartir las bases gramaticales, debido a que la falta de ellas dificultaba a los estudiantes el abordaje de ciertas tareas que requerían su manejo. Por su parte, el tutor Elí expresó que se dio cuenta de que a uno de los alumnos que asesoró le costaba identificar las partes de la oración y, por tratarse de algo muy básico de la gramática, limitaba su progreso general en la redacción (ETEE 8:2).

Por lo anterior, se deduce que es muy difícil lograr atender las necesidades de escritura cuando los alumnos ni siquiera conocen lo más básico de la gramática.

Recursos. Los tutores ofrecieron recursos a los alumnos para lograr que estos se convirtieran en escritores independientes. El tutor es como una especie de entrenador, pues ayuda a alcanzar los objetivos utilizando recursos de forma eficaz (Gómez-Collado, 2012). Ruth señaló que su tutora le dio un formato en el que podía anotar el título, la bibliogra- fía y el resumen de los artículos que leía (EAR 1:18). Al respecto, Noemí dijo que le había ofrecido esa tabla para que pudiera evitar el plagio, el cual constituía una de las principales fuentes de temor manifestada por Ruth (ETPN 5:10), quien también dijo que Noemí le había dado otros recursos para identificar los diferentes tipos de nexos (EAR 1:18). El tutor Elí mencionó que es complicado abarcar mucho en una hora y que es mejor darles ideas y recursos donde los alumnos puedan buscar y convertirse en sus propios críticos de escritura (ETEE $8: 12)$.

Valoración de las tutorías. Se encontraron diversas valoraciones de las tutorías, entre ellas las de (a) mejoría en la escritura, (b) ambiente de confianza, (c) disposición del tutor y (d) preferencia del alumno de un seguimiento con el mismo tutor. Se discuten a continuación.

Mejoría en la escritura. Los alumnos y tutores mencionaron que percibieron mejoría en la escritura y que se fueron resolviendo las necesidades iniciales, lo cual les causó satisfacción y fue un motivo para continuar las tutorías, sobre todo con el mismo tutor (ETPN 5:12). Ruth mencionó que había mejorado en su escritura y que, aunque había dejado de asistir a las tutorías, aplicaba de manera independiente lo que su tutora le había enseñado (EAR 1:11). De igual manera, Miriam reveló que sentía que, gracias a la tutoría, iba a entregar un ensayo de calidad en comparación con otros y que sentía mejoría en su escritura (EAM 2:15).

Se observó que tanto tutores como alumnos percibieron mejorías en la escritura, lo cual les generaba satisfacción y mayor motivación para continuar con las tutorías. Jesús, por ejemplo, señaló 
que le encantaba trabajar con Juan, porque veía mucho avance y que observar que había producción le causaba motivación (ETEJ 7:26).

Ambiente de confianza. Los alumnos mencionaron sentirse más cómodos con los tutores pares. Fuchs et al. (1997) afirman que esta modalidad brinda libertad al tutor, anima al estudiante a realizar preguntas que no se atrevería hacerle al profesor y que "puede incrementar el rendimiento más que la instrucción tradicional" (p. 270).

Miriam, quien tuvo una tutora par, manifestó que, como su tutora también era alumna, sintió que congeniaron bien y que no se podría haber expresado tanto si hubiera sido un tutor mayor (EAM $2: 11)$. Este tipo de modalidad, gracias al contacto personalizado, la confianza y la empatía entre ambos actores, "conduce a una mayor apertura para conversar de los problemas de escritura y por lo tanto llegar juntos a descubrir las estrategias más convenientes" (Molina Natera, 2011; Moliner-Miravet et al., 2014). Llanes et al. (2017) añaden que estas acciones refuerzan también el aprendizaje en los tutores, quienes crecen en ámbitos tanto sociales como profesionales y escriturales (Chois-Lenis et al., 2017).

Al tener edades similares y experiencias cercanas a la escritura, el tutor par y el alumno pueden congeniar y resolver juntos las dificultades, sin olvidar que el alumno es el autor y que de él dependen las decisiones para mejorar el escrito, de manera que conserve su estilo y autoría (Carlino, 2008). Al respecto, Noemí mencionó que su alumna

se sintió en confianza, ya que había esa libertad de que ella pudiera externar sus preguntas y yo fui muy honesta con ella al decirle que también estaba aprendiendo a la vez con ella.
Entonces, creo que esa confianza ayudó a que se sintiera motivada y también a ver avances. (ETPN 5:11) No obstante, se observó que no solo los tutores pares pueden establecer lazos de confianza con los alumnos, sino que también los tutores de experiencia. Tal fue el caso de Juan y su tutor de experiencia Jesús, quienes expresaron haber establecido grandes lazos de confianza. Así lo afirmó Juan, el alumno, al decir:

Aumentaba mi confianza, porque no solo me veía como un sujeto de prueba del taller, sino me veía como una persona a la cual en verdad quiere ayudar. Porque yo creo que es algo que nos caracteriza ... que no podemos dejar de ver al alumno como persona, que no podemos dejar de preguntarle "oye sabes qué, cómo te va, como estás", cosas así. Al contrario, en vez de molestarme me daba... esa confianza de abrirme con él y daba un ambiente muy bonito dentro del salón. (EAJ 4:17)

En síntesis, independientemente del nivel de experiencia o edad, se percibieron lazos de confianza entre tutores y alumnos.

Disposición del tutor. En las tutorías de escritura el trabajo es personalizado y dirigido a atender las necesidades del alumno (Harris, 1995). Se observó que los alumnos se sintieron satisfechos con las tutorías gracias a la disposición que percibieron por parte de su tutor.

Ruth, por ejemplo, manifestó que valoró la disposición de Noemí, quien traía más ideas y buscaba maneras para que ella pudiera aprender (EAR 1:18). $\mathrm{Su}$ percepción concuerda con lo afirmado por Miriam acerca de su tutora, de quien se expresó como una persona amable, eficaz, que la ayudó a prosperar y a cumplir sus metas (EAM 2:21). Esto 
armoniza con Molina Natera (2016), quien señala que un tutor de escritura es respetuoso y tiene disposición para escuchar y atender las necesidades de los alumnos.

Se observó también que algunos tutores ofrecían tutorías fuera de horario o, incluso, dedicaban más tiempo que el asignado a sus tutorías. Todos mostraron una actitud positiva y dispuesta. El perfil de un buen tutor se destaca por la disposición que tiene para ayudar, escuchar, crear un ambiente de confianza y atender las necesidades individuales del alumno. Las tutorías no son replicables, pues se adaptan a cada alumno; sin embargo, la disposición del tutor propicia un mejor ambiente para el aprendizaje de la escritura.

Seguimiento con el mismo tutor. Se encontró que los alumnos preferían continuar con el mismo tutor, porque sentían que estos ya conocían sus necesidades y podían llevar un seguimiento (EAR 1:10). Jesús expresó que creía que Juan deseaba continuar con las tutorías porque se habían "enganchado" con un proyecto en particular y, como ya llevaban cierto avance, deseaban darle seguimiento y terminarlo (ETEJ 7:46). Se observó que, en su mayoría, los alumnos que volvían solicitaban al mismo tutor, porque podía brindarles un seguimiento de su progreso en la escritura.

Retos de las tutorías. Los principales retos identificados fueron (a) la falta de tiempo, (b) la limitación del tutor, (c) la brecha generacional, (d) la evaluación y (e) la difusión.

Falta de tiempo. Se observó que la mayoría de los alumnos presentaban dificultades para acudir a las asesorías debido a la falta de tiempo, ya que la mayoría trabajaba. Además, preferían utilizar sus tiempos libres en las diversas actividades que ofrece la universidad. Miriam, quien solo pudo asistir una vez a las tutorías, expresó que sería mejor "que hubiera horarios un poco más accesibles pensando en los que trabajan" (EAM 2:24). Valeria simplemente dijo que había dejado de ir a las tutorías porque no tenía tiempo (EAR :15).

Samuel sugirió habilitar los fines de semana para tutorías, pues la mayoría de los jóvenes lo dedican para estudiar, pero finalmente dijo que hace falta más interés por parte de los alumnos y que, dentro de sus limitaciones, deberían aprovechar las tutorías de escritura (EAS 3:7). Juan mencionó también las restricciones de los horarios de los alumnos, pero también de los tutores, pues no hay mucha variedad ni flexibilidad. Sin embargo, concluyó también en que, si los alumnos saben que tienen necesidades, deben sacrificar unas cosas por otras (EAJ 4:5).

Se observó que los tutores tienen mucha carga de trabajo, por lo que les resulta difícil adecuar sus horarios de atención al de los alumnos. Regularmente los tutores con más experiencia no ofrecen muchas asesorías y, además, están fuera del alcance de los alumnos.

Los hallazgos de esta investigación destacan la falta de tiempo como el mayor reto de las tutorías de escritura. Esto coincide con lo observado por Molina Natera (2015), quien expresa que uno de los principales retos de las tutorías es el tiempo limitado para las tutorías presenciales. Además de los aspectos mencionados, es necesario observar que los alumnos mayormente acuden a una sola asesoría, en la que es imposible cubrir sus necesidades de escritura.

Limitación del tutor. Diversas investigaciones apuntan a que el acompañamiento de los tutores pares constituye 
la mejor estrategia para los centros de escritura (Calle Arango et al., 2017; Llanes et al., 2017; Molina Natera, 2014), siempre y cuando se capacite constantemente al tutor (Chois-Lenis et al., 2017). En este estudio, Noemí dijo haber disfrutado su experiencia, pero que no se sentía muy capacitada como tutora (ETPN 5:20); al igual que Jocabet, quien manifestó sentir limitaciones en las diferentes disciplinas a las que pertenecen los alumnos (ETPJ 6:39).

Sin embargo, Molina Natera (2011) afirma que esto desarrolla en el tutor la "sensibilidad para ayudar a los demás; aprendizaje de otras temáticas y disciplinas, pues generalmente deben trabajar con personas de otras carreras" (p. 4). El tutor Jesús dijo que, cuando acudía a él un alumno de un área que no era la suya, se sentía limitado, pues desconocía el contenido de los textos, lo que le producía frustración, pues no podían avanzar tanto (ETEJ 8:22). Por otro lado, Elí mencionó que las diferentes disciplinas no deberían significar un problema, porque la tutoría consiste en revisar la redacción $\mathrm{y}$, si surge alguna duda sobre algún término, basta con preguntarle al alumno (ETEE 8:9).

Brecha generacional. Los tutores pares y de experiencia coincidían en las reuniones quincenales donde compartían sus dudas y experiencias. Sin embargo, Jocabet expresó sentirse insegura al comparar sus conocimientos con los de sus colegas con más experiencia:

Es que yo también me siento un poquito así, menos, no sé, porque siento que todos mis compañeros asesores, pues son doctores... Entonces siento que ya... saben un montón y yo siento que no sé tanto como ellos. (ETPJ 6:38)

Se observó que los tutores pares eran quienes menos participaban y los tuto- res con experiencia eran quienes dominaban las conversaciones, ponencias y experiencias. Esto coincide con lo que expresan Fernández Pequeño y Ulloa Hung (2008), respecto de que la brecha generacional trae consigo "consecuencias distintas y desmesuradas" (p. 53). En este sentido, si el tutor no está lo suficientemente preparado y no se siente capacitado, interferirá en la comunicación y en su desempeño.

El reto de la brecha generacional entre los tutores de experiencia, los tutores pares y los alumnos mostró relación con la ausencia de bases gramaticales entre los dos últimos grupos, pues el nivel lingüístico de los tutores de experiencia está muy por encima de ellos. Molina Natera (2012) sugiere que se llegue a un punto intermedio respecto de esta brecha existente entre los tutores experimentados en la escritura y los alumnos menos expertos.

Evaluación. La evaluación produjo reacciones diferidas. Mientras que Miriam la consideró una pérdida de tiempo (EAM 2:2), Ruth expresó que deseaba una evaluación continua (EAR 1:19). Por su parte, Juan expresó que no se imaginó que le harían una prueba al inicio de su asesoría y que no estaba preparado para hacerla, por lo que no se sintió cómodo (EAJ 4:6). Sin embargo, Samuel dijo que fue una grata sorpresa para él, pero que nunca le fueron revelados sus resultados, lo que consideró que debería mejorarse (EAS 3:28).

Se observó cierta resistencia hacia la evaluación por parte de la mayoría, pues los alumnos preferían que los tutores se enfocaran en el texto y sentían que perdían tiempo, debido a que invertían la mitad de la asesoría en la evaluación inicial. Los hallazgos respecto de la evaluación resultan importantes. 
Molina Natera (2019a) afirmó que las tutorías de escritura no derivan en notas, calificaciones o puntajes. Por lo tanto, las evaluaciones mediante exámenes o pruebas no concuerdan con las premisas de las tutorías de escritura. Sin embargo, algunos alumnos manifestaron su deseo por evaluaciones rápidas para saber con exactitud sus dificultades y deseaban una evaluación continua.

Difusión. La mayoría de los entrevistados expresó que se desconoce el servicio de las tutorías de escritura y que quienes lo conocen no saben exactamente su propósito, pues esperan solo una edición del texto, lo más rápida posible, por el tutor de escritura.

Juan mencionó que la mayoría de sus compañeros no sabían del proyecto y que debía ser compartido "dentro de la comunidad estudiantil con más fuerza", ya que "faltó difusión continua" (EAJ 4:11). Samuel coincidió al respecto y agregó que también se debería comunicar a los diferentes centros de investigación de la universidad para que sepan que hay un espacio en el que se brinda acompañamiento de escritura académica (EAS 3:9). Se observó poco esfuerzo en difundir las tutorías de escritura. Pocos alumnos y docentes saben de este servicio gratuito disponible en la universidad.

\section{Conclusiones}

Al ser un centro de escritura novel, se necesita más capacitación sobre los propósitos de las tutorías de escritura, no solo a los que ofrecen el servicio, sino a toda la comunidad universitaria, pues algunos acuden buscando en el tutor una firma de aprobación o edición del documento, lo cual va en contra de los principios de las tutorías de escritura.

Según los resultados de este estudio, se pueden identificar aspectos positivos de las tutorías de escritura. Sin embargo, para comprender la poca demanda de estudiantes que buscan las asesorías, debe considerarse la falta de tiempo como el mayor reto de las tutorías de escritura. En una institución donde una gran parte de los estudiantes trabaja a la par de sus estudios, se debe analizar con profundidad la compatibilidad de horarios entre los tutores y alumnos debido a la carga de estudio y al trabajo de ambos.

Una vez llevadas a cabo las adecuaciones en las áreas de mejora, se debe realizar una estrategia de difusión del servicio, de tal manera que un mayor número de alumnos y maestros conozcan los beneficios que ofrecen las tutorías de escritura y las soliciten con regularidad.

\section{Referencias}

Aguirre Seura, L. (2016). Evaluación de una propuesta para el desarrollo de la escritura en estudiantes universitarios a partir de habilidades de metacognición. Logos: Revista de Lingüistica, Filosofía y Literatura, 26(2), 181-196. https://doi.org/10.15443/RL26015

Ballesteros Pérez, D. V. (2016). El lenguaje escrito como canal de comunicación y desarrollo humano. Razón y Palabra, 20(2_93), 442-455. https://www.revistarazonypalabra.org/index.php/ryp/article/view/27

Calle Álvarez, G. Y. (2014). La habilidad del pensamiento crítico para el establecimiento de una posición frente a un tema en la escritura digital. Zona Próxima, 21, 17-32. https://doi.org/10.14482/zp.21.5953

Calle Arango, L., Pico, A. L. y Murillo, J. H. (2017). Los centros de escritura: entre nivelación académica y construcción de conocimiento. Cadernos de Pesquisa, 47(165), 872-895. https://doi. org $/ 10.1590 / 198053143882$

Carlino, P. (2004). Escribir a través del currículo: tres modelos para hacerlo en la universidad. Lectura y Vida. Revista Latinoamericana de Lectura, 25(1), 16-27. http://www.lecturayvida.fahce.unlp.edu.ar/ numeros/a25n1/25_01_Carlino.pdf 
Carlino, P. (2008). Revisión entre pares en la formación de posgrado. Lectura y Vida, 29(2), 20-28. http:// www.lecturayvida.fahce.unlp.edu.ar/numeros/a29n2/29_02_Carlino.pdf

Cassany, D. (1993). La cocina de la escritura. Anagrama.

Cassany, D. (1994). Enseñar lengua. Graó.

Cassany, D. (1995). Describir el escribir: cómo se aprende a escribir. Paidós.

Castro Azuara, M. A. y Sánchez Camargo, M. (2015). Escribir en la universidad: la organización retórica del género tesina en el área de humanidades. Perfiles Educativos, 37(148), 50-67. https://doi. org/10.1016/j.pe.2015.11.007

Chois-Lenis, P. M., Casas-Bustillo, A. C., López-Higuera, A., Prado-Mosquera, D. M. y Cajas-Paz, E. Y. (2017). Percepciones sobre la tutoría entre pares en escritura académica. Magis: Revista Internacional de Investigación en Educación, 9(19), 165-184. https://doi.org/10.11144/Javeriana.m9-19.ptpe

Creswell, J. W. (2012). Educational research: Planning, conducting, and evaluating. Pearson.

Diario Oficial de la Federación. (2016). Ley Federal de Protección de Datos Personales en Posesión de los Particulares. http://www.diputados.gob.mx/LeyesBiblio/pdf/LFPDPPP.pdf

Ferreira, A., Salcedo, P., Kotz, G. y Barrientos, F. (2012). La arquitectura de ELE-TUTOR: un sistema tutorial inteligente para el español como lengua extranjera. Revista Signos, 45(79), 102-131. https:// doi.org/10.4067/S0718-09342012000200001

Flores Guerrero, D. (2016). La importancia e impacto de la lectura, redacción y pensamiento crítico en la educación superior. Zona Próxima, 24, 128-135. https://rcientificas.uninorte.edu.co/index.php/zona/ article/view/7200

Fuchs, D., Fuchs, L. S., Mathes, P. G. y Simmons, D. C. (1997). Peer-assisted learning strategies: Making classrooms more responsive to diversity. American Educational Research Journal, 34(1), 174-206. https://doi.org/10.3102/00028312034001174

Giraldo Giraldo, C. (2015). La escritura en el aula como instrumento de aprendizaje. Estudio en universidades. Ánfora, 22(38), 39-58. https://doi.org/10.30854/anf.v22.n38.2015.25

Gómez-Collado, M. E. (2012). La percepción de los estudiantes sobre el Programa de Tutoría Académica. Convergencia: Revista de Ciencias Sociales, 58, 209-233. https://convergencia.uaemex.mx/article/ view/1103

González Jaimes, E. I., Hernández Prieto, M. L. y Márquez Zea, J. (2013). La oralidad y la escritura en el proceso de aprendizaje: aplicación del método aprende a escuchar, pensar y escribir. Contaduría y Administración, 58(2), 261-278. https://doi.org/10.1016/S0186-1042(13)71217-8

Guzmán-Simón, F. y García-Jiménez, E. (2014). Los hábitos lectoescritores en los alumnos universitarios. Revista Electrónica Interuniversitaria de Formación del Profesorado, 17(3), 79-92. https://doi. org/10.6018/reifop.17.3.204071

Harris, M. (1995). Talking in the middle: Why writers need writing tutors. College English, 57(1), 27-42. https://library.ncte.org/journals/ce/issues/v57-1/9147

Hobson, E. H. (1992). Writing the writing center. USU Press.

Llanes, J., Quiles-Fernández, E. y Noguera, E. (2017). La tutoría entre iguales: una experiencia del grado de pedagogía de la Universidad de Barcelona. REDU: Revista de Docencia Universitaria, 15(2), 229244. https://doi.org/10.4995/redu.2017.7126

Merriam, S. B. (1998). Qualitative research and case study applications in education. Jossey-Bass.

Merriam. S. B. (2009). Qualitative research: A guide to design and implementation (2a ed.). Jossey-Bass.

Merrian, S. B. y Tisdell, E. J. (2016). Qualitative research: A guide to design and implementation. JosseyBass.

Molina Natera, V. (2011, 15-17 de junio). Tutorías entre pares: el caso del Centro de Escritura Javeriano [Presentación de ponencia]. VI Congreso Internacional de la Cátedra UNESCO para el Mejoramiento de la Calidad y Equidad de la Educación en América Latina con base en la Lectura y Escritura, Barranquilla, Colombia.

Molina Natera, V. (2012). Tensiones entre discursos de estudiantes y profesores universitarios sobre la lectura y la escritura. Signo y Pensamiento, 31(61), 126-141. https://doi.org/10.11144/Javeriana.syp3161.tede

Molina Natera, V. (2014). Centros de escritura: una mirada retrospectiva para entender el presente y futuro de estos programas en el contexto latinoamericano. Legenda, 18(18), 9-33. http://erevistas.saber.ula. ve/index.php/legenda/article/view/5205

Molina Natera, V. (2015). Panorama de los centros y programas de escritura en Latinoamérica. Santiago de Cali: Pontificia Universidad Javeriana Cali. http://vitela.javerianacali.edu.co/handle/11522/8091 Molina Natera, V. (2016). Los centros de escritura en latinoamérica: consideraciones para su diseño e 


\section{GRANADOS AYALA}

implementación. En G. Bañales Faz, M. Castelló Badía y N. A. Vega López (Coords.), Leer y escribir en la educación superior: propuestas educativas basadas en la investigación (pp. 339-362). Asesoría en Tecnologías y Gestión Educativa.

Molina Natera, V. (2019a). El discurso pedagógico en las tutorías de escritura: develando elementos de una práctica educativa. Revista Mexicana de Investigación Educativa, 24(80), 125-148. http://www.comie. org.mx/revista/v2018/rmie/index.php/nrmie/article/view/1240

Molina Natera, V. (2019b, 12-14 de junio). La tutoría de escritura para el desarrollo de escritores autónomos [Presentación de Ponencia]. IV Congreso Internacional de Lectura y Escritura en la Sociedad Global, Monterrey, Nuevo León, México.

Moliner-Miravet, L., Sales-Ciges, A. y Moliner-García, O. (2014). An experience of reciprocal peer tutoring at the university. Procedia - Social and Behavioral Sciences, 116, 2809-2812. https://doi.org/10.1016/j. sbspro.2014.01.661

Moyano, E. (2010). Escritura académica a lo largo de la carrera: un programa institucional. Revista Signos, 43(74), 465-488. https://doi.org/10.4067/S0718-09342010000500004

Murray, D. H. (1982). Learning by teaching. Boyton/Cook.

Patton, M. Q. (2015). Qualitative research and evaluation methods (4a ed.). Sage.

Piaget, J. (1972). A dónde va la educación. Teide.

Sagor, R. (2000). Action research. Association for Supervision and Curriculum Development.

Schunk, D. H. (2012). Teorías del aprendizaje: una perspectiva educativa (6a ed.). Pearson.

Vygotsky, L. S. (1995). Pensamiento y lenguaje: teoría del desarrollo cultural de las funciones psíquicas. Fausto.

Recibido: 26 de agosto de 2021

Revisado: 28 de septiembre de 2021

Aceptado: 10 de octubre de 2021 